\title{
Comparison of human chorionic gonadotropin $+\beta$ and invasive trophoblast antigen disappearance rates in serum after evacuation of molar pregnancy
}

\author{
NIENKE E. VAN TROMMEL ${ }^{1,2}$, FRED C.G.J. SWEEP ${ }^{1}$, H. ALEC ROSS ${ }^{1}$, \\ LEON F.A.G. MASSUGER ${ }^{2}$ and CHRIS M.G. THOMAS ${ }^{1,2}$ \\ Departments of ${ }^{1}$ Chemical Endocrinology, ${ }^{2}$ Obstetrics and Gynecology, \\ Radboud University Nijmegen Medical Centre, P.O. Box 9101, 6500 HB Nijmegen, The Netherlands
}

Received April 18, 2006; Accepted June 28, 2006

\begin{abstract}
After the evacuation of a hydatidiform mole, the spontaneous regression or the persistent trophoblastic disease (PTD) needing chemotherapy, is monitored by determining the serum human chorionic gonadotropin (hCG) concentration. Hyperglycosylated hCG (invasive trophoblast antigen, ITA) has been suggested to be of clinical value in the diagnosis and follow-up of gestational trophoblastic disease including PTD. To further document the relationship between ITA and hCG in spontaneous post-molar regression and during chemotherapy treatment of PTD, we used distinct immunoassays to measure the concentrations of $h C G+\beta$ and ITA in serum from three groups of patients after the evacuation of moles. For each group [uneventful post molar hCG regression, group 1; PTD treated with Methotrexate (MTX) (monochemotherapy), group 2; and PTD with MTX and polychemotherapy (EMA-CO), group 3], we compared the time course of the serum concentrations after evacuation, and determined the disappearance rates (half-lives) within and between treatment groups. Significantly longer mean serum half-lives for $\mathrm{hCG}+\beta$ and ITA were found in the polychemotherapy (group 3: 3.02 and 2.51 weeks) as compared to the mono-chemotherapy group (group 2: 0.96 and 0.90
\end{abstract}

Correspondence to: Dr Chris M.G. Thomas, Department of Chemical Endocrinology, 479 ACE, Radboud University Nijmegen Medical Centre, PO Box 9101, 6500 HB Nijmegen, The Netherlands

E-mail: c.thomas@ace.umcn.nl

Abbreviations: hCG, human chorionic gonadotropin; PTD, persistent trophoblastic disease; ITA, invasive trophoblast antigen; MTX, Methotrexate; EMA-CO, multi-agent chemotherapy; GTD, gestational trophoblastic disease

Key words: human chorionic gonadotropin, invasive trophoblast antigen, hydatidiform mole, persistent trophoblastic disease, radioimmunoassay, chemotherapy, half-life weeks) and the uneventful regression group (0.81 and 0.66 weeks) (each, $\mathrm{p}=0.003$ ), but no differences were observed between the mono-chemotherapy and the uneventful regression group. Significantly shorter mean half-lives for ITA than those calculated for $\mathrm{hCG}+\beta$ were observed in all three groups of patients. The implication and the possible clinical value of the more rapid regression of ITA to baseline levels as compared to $h C G+\beta$ remain to be investigated prospectively.

\section{Introduction}

The glycoprotein hormone human chorionic gonadotropin (hCG) is produced by trophoblastic tissue and therefore is a key marker in pregnancy and gestational trophoblastic disease (GTD). The disappearance of serum hCG concentrations is monitored by constructing a hCG regression curve after mole evacuation (1). A treated molar pregnancy may persist and may either lead to persistent trophoblastic disease (PTD) or to the development of choriocarcinoma. The reported frequency of PTD is $15-20 \%$ in complete hydatidiform moles (2), 4-9\% in partial hydatidiform moles $(3,4)$, while choriocarcinoma occurs less frequently. In order to prevent complications from metastatic disease, PTD and choriocarcinoma need to be treated with chemotherapy. Once diagnosed and classified, low-risk PTD is typically treated with single agent therapy (Methotrexate or Actinomycin-D) $(5,6)$ while high-risk PTD is most widely treated with combination therapy (EMA-CO: Etoposide, MTX, Actinomycin-D, Cyclophos-phamide and Vincristine) (5). PTD is recognised by plateauing, or even increasing levels of hCG in three subsequent weekly blood samples, with one or more hCG values above the 95th percentile of the hCG-regression curve (1).

The glycoprotein hormone hCG $\left(\mathrm{M}_{\mathrm{r}} 37,500\right)$ is composed of two non-covalently bound subunits, $\alpha$ and $\beta$, with molecular weight of 14,000 and 23,500, respectively. The $\alpha$ subunit of hCG is homologous with that of the heterodimeric pituitary glycoprotein hormones LH, FSH and TSH. The ßchains distinguish these hormones from each other and they determine the biological activity. Eight carbohydrate chains are attached to hCG and comprise about one-third of the molecular mass of intact hCG, two N-linked carbohydrate 

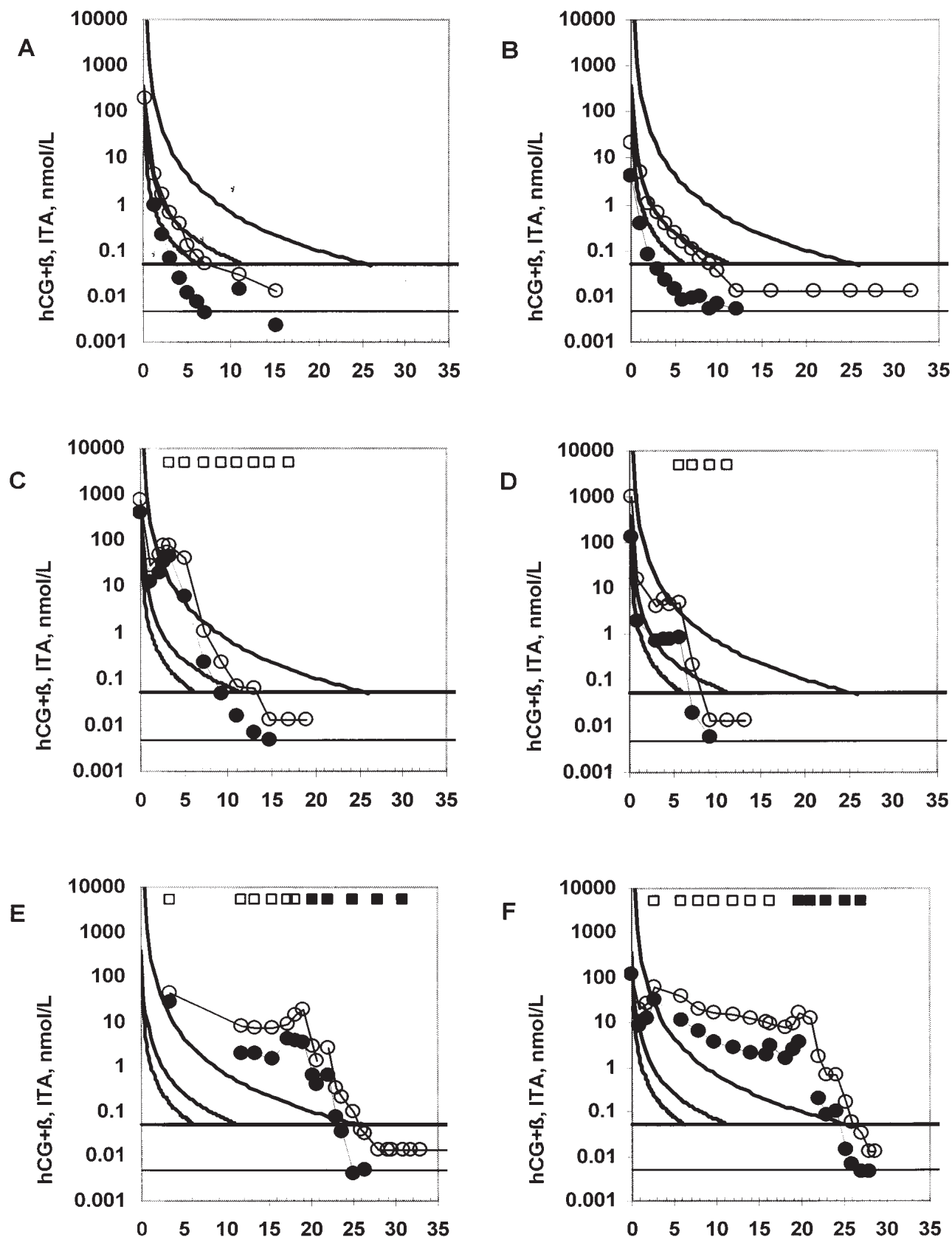

weeks after evacuation

weeks after evacuation

Figure 1. Serum concentrations of $\mathrm{hCG}+\beta$ (solid line with $\mathrm{O}$ ) and ITA (solid line with $\bullet$ ) after evacuation of molar pregnancy followed by spontaneous regression (A, B), or by persistent trophoblastic disease, treated either with first-line chemotherapy (MTX) (C, D, $\square$ ), or with MTX ( $\square$ ) followed by secondline chemotherapy (EMA-CO) $(\mathrm{E}, \mathrm{F}, \boldsymbol{\square})$. The three solid lines $(*)$ represent the 5 th, 50 th, and the 95 th percentile of the uneventful regression of hCG+ß. The 95th percentile of the reference interval of healthy control pregnancies for the hCG+ $\beta$ assay (fat line) was established at $0.053 \mathrm{nmol} / \mathrm{l}$ ( $18.6 \mathrm{IU} / 1$ of the WHO third IS hCG 75/537). Because the 95th percentile of the reference interval of healthy control pregnancies for the ITA assay was not established, the analytical sensitivity of the ITA assay $(<0.005 \mathrm{nmol} / \mathrm{l})$ is indicated (solid line). Conversion factors used are hCG+ß: $1 \mu \mathrm{g} / 1$, equivalent to $9.29 \mathrm{IU} / 1$, equivalent to 0.0267 nmol/1; ITA: $1 \mu \mathrm{g} / 1$, equivalent to $0.0238 \mathrm{nmol} / 1$.

chains are attached to the hCG $\alpha$-subunit, two others to the hCG ß-subunit, while four O-linked sugar side chains are attached to hCGß. Whereas normal and molar pregnancies show only limited proportions of more complex triantennary $\mathrm{N}$-linked oligosaccharides $(0-30 \%)$ and larger hexasaccharide-type-O-linked carbohydrates (0-20\%), choriocarcinoma consists of up to $100 \%$ of these larger $\mathrm{N}$ - and Olinked oligosaccharides called hyperglycosylated hCG (7).
In normal pregnancy, hyperglycosylated hCG is gradually replaced by hCG. Hyperglycosylated hCG $\left(\mathrm{M}_{\mathrm{r}} 42,000\right)$ accounts for $>80 \%$ of total hCG in the first week, $50 \%$ in the third week after implantation, while in the third trimester it diminishes to $2 \%$ (8). Kovalevskaya et al showed that hyperglycosylated hCG is produced in placental stem cells or cytotrophoblasts and that the less glycosylated hCG form is synthesized mainly in syncytiotrophoblasts (9). Hyper- 
Table I. Treatment characteristics of patients after evacuation of molar pregnancies: Observation period, number of specimens tested (no.), concentration ranges of the two compared analytes, and number (n) and time period (start-end) of the chemotherapy courses.

\begin{tabular}{|c|c|c|c|c|c|c|}
\hline Group & Case & $\begin{array}{c}\text { Period } \\
\text { weeks } \\
\text { (min-max) } \\
\text { nmol/l }\end{array}$ & $\begin{array}{c}\mathrm{hCG}^{+} \beta, \\
\text { no. } \\
(\min -\max ) \\
\mathrm{nmol} / 1\end{array}$ & $\begin{array}{c}\text { ITA } \\
\text { no. } \\
\text { n (start-end) } \\
\text { weeks }\end{array}$ & $\begin{array}{c}\text { Single-agent CT } \\
\text { MTX } \\
\text { n (start-end) } \\
\text { weeks }\end{array}$ & $\begin{array}{c}\text { Multi-agent CT } \\
\text { EMA-CO } \\
\text { n (start-end) } \\
\text { weeks }\end{array}$ \\
\hline \multirow[t]{7}{*}{1} & 1 & 15 & $10(<0.027-4.8)$ & $9(<0.005-0.95)$ & none & none \\
\hline & 2 & 32 & $14(<0.027-2.6)$ & $6(<0.005-0.81)$ & none & none \\
\hline & 3 & 20 & $9(<0.027-4.5)$ & $7(<0.005-1.1)$ & none & none \\
\hline & 4 & 29 & $15(<0.027-3.5)$ & $12(<0.005-0.33)$ & none & none \\
\hline & 5 & 11 & $11(<0.027-4.0)$ & $11(<0.005-0.60)$ & none & none \\
\hline & 6 & 32 & $17(<0.027-5.1)$ & $11(<0.005-0.39)$ & none & none \\
\hline & 7 & 95 & $11(<0.027-2.9)$ & $6(<0.005-0.93)$ & none & none \\
\hline \multirow[t]{7}{*}{2} & 1 & 93 & $16(<0.027-75)$ & $10(<0.005-15)$ & $7(5-16)$ & none \\
\hline & 2 & 66 & $23(<0.027-75)$ & $10(<0.005-45)$ & $8(3-17)$ & none \\
\hline & 3 & 13 & $9(<0.027-16)$ & $7(<0.005-1.9)$ & $4(6-11)$ & none \\
\hline & 4 & 59 & $18(<0.027-4.3)$ & $6(<0.005-1.1)$ & $6(11-19)$ & none \\
\hline & 5 & 73 & $21(<0.027-29)$ & $9 \quad(0.007-24)$ & $9(3-19)$ & none \\
\hline & 6 & 24 & $9(<0.027-12)$ & $8(<0.005-1.4)$ & $4(4-12)$ & none \\
\hline & 7 & 68 & $17(<0.027-6.7)$ & $10 \quad(0.006-1.4)$ & $3(12-18)$ & none \\
\hline \multirow[t]{6}{*}{3} & 1 & 129 & $37(<0.027-290)$ & $19(<0.005-86)$ & $7(5-21)$ & $4(23-32)$ \\
\hline & 2 & 38 & $22(<0.027-43)$ & $14(<0.005-27)$ & $6(3-18)$ & $5(20-31)$ \\
\hline & 3 & 61 & $17(<0.027-590)$ & $16(<0.005-110)$ & $10(5-23)$ & $1(25)$ \\
\hline & 4 & 41 & $34(<0.027-250)$ & $32(<0.005-110)$ & $9(4-19)$ & $3(24-29)$ \\
\hline & 5 & 73 & $24(<0.027-24)$ & $13(<0.005-13)$ & $7(6-19)$ & $3(21-34)$ \\
\hline & 6 & 95 & $37(<0.027-61)$ & $20(<0.005-31)$ & $7(3-16)$ & $5(20-27)$ \\
\hline
\end{tabular}

Group 1, uneventful regression after evacuation of the molar pregnancy; group 2, persistent trophoblastic disease after evacuation of the molar pregnancy and treatment with single-agent chemotherapy (MTX); group 3, persistent trophoblastic disease after evacuation of the molar pregnancy and treatment with multiagent chemotherapy (EMA-CO) following MTX.

glycosylated hCG is produced by invasive or cancerous trophoblast cells in choriocarcinoma. This suggests that hyperglycosylated hCG is a product of separate trophoblast cells possibly synthesized in invasive trophoblast cells, for this reason hyperglycosylated hCG is also referred to as invasive trophoblast antigen (ITA) (8).

It has been suggested that ITA is of clinical value in the diagnosis and follow-up of GTD (10). In women with persistently low levels of hCG following evacuation without signs of GTD, the ITA accounts for $<25 \%$ of total $\mathrm{hCG}$ immunoreactivity (11). ITA is considered to be effective in detecting invasive trophoblastic disease in patients who experienced a sudden steep rise in their serum hCG concentrations after years of persistent low serum hCG concentrations because ITA then accounts for $81-100 \%$ of the highly increased total hCG concentration (11). However, it was not explicitly stated that the determination of ITA is able to exclude the presence of invasive trophoblastic disease in the reported cases with persistently low levels of hCG without signs of PTD. To further document the relationship between ITA and hCG in PTD, we compared the time course and disappearance rate (half-life) in serum of ITA with those of already available $\mathrm{hCG}+\beta$ data determined longitudinally in serum from three groups of patients following the evacuation of moles. These groups were comprised of uneventful post molar hCG regression, management of PTD with MTX (mono-chemotherapy), and management of PTD with MTX and poly-chemotherapy (EMA-CO).

\section{Materials and methods}

Patients. The patients participating in the present study gave an informed consent before registration at the Dutch Central Registry for Hydatidiform Moles located at the Radboud University Nijmegen Medical Centre (RUNMC). After collection, blood samples were centrifuged and serum was sent to our institute where it was assayed for total hCG and kept at $-20^{\circ} \mathrm{C}$ until further analysis. From this database, we randomly selected 20 patients who were all registered between 2000 and 2002, and from whom longitudinally collected serum samples were available for ITA analysis. Of these 20 patients, 7 had a hydatidiform mole with uneventful hCG regression after evacuation, as derived from the normal hCG regression curve (1). Thirteen patients with a 
Table II. Mean (min-max) half-lives $\left(\tau_{1 / 2}\right)$ of hCG+ß and ITA after evacuation of hydatidiform moles followed by spontaneous regression, mono-chemotherapy (MTX), or poly-chemotherapy (EMA-CO) following MTX.

\begin{tabular}{|c|c|c|c|c|c|c|}
\hline & $\begin{array}{c}\text { Group 1 } \\
\text { Uneventful } \\
\text { regression (PTD-) }\end{array}$ & $\begin{array}{c}\text { Group } 2 \\
\text { Mono-chemotherapy } \\
\text { (PTD+MTX) }\end{array}$ & $\begin{array}{c}\text { Group } 3 \\
\text { Poly-chemotherapy } \\
\text { (PTD+MTX+EMA-CO) }\end{array}$ & $\begin{array}{l}\text { Significance }^{\mathrm{a}} \\
\text { Group1 vs } 2 \\
\mathrm{p}\end{array}$ & $\begin{array}{c}\text { Significance }^{\mathrm{a}} \\
\text { Group } 1 \text { vs } 3 \\
\text { p }\end{array}$ & $\begin{array}{c}\text { Significance }^{\mathrm{a}} \\
\text { Group } 2 \text { vs } 3 \\
\mathrm{p}\end{array}$ \\
\hline Number of cases & 7 & 7 & 6 & & & \\
\hline $\begin{array}{l}\tau_{1 / 2} \text { of hCG+ } \beta \\
\text { (weeks) }\end{array}$ & $\begin{array}{c}0.81 \\
(0.39-1.4)\end{array}$ & $\begin{array}{c}0.96 \\
(0.66-1.4)\end{array}$ & $\begin{array}{c}3.02 \\
(2.2-3.9)\end{array}$ & NS & 0.003 & 0.003 \\
\hline $\begin{array}{l}\tau_{1 / 2} \text { of ITA } \\
\text { (weeks) }\end{array}$ & $\begin{array}{c}0.66 \\
(0.27-1.0)\end{array}$ & $\begin{array}{c}0.90 \\
(0.57-1.2)\end{array}$ & $\begin{array}{c}2.51 \\
(1.5-3.1)\end{array}$ & NS & 0.003 & 0.003 \\
\hline Significance $^{b} \quad p$ & & & & & & \\
\hline$\tau_{1 / 2}$ of hCG $+\beta$ vs ITA & 0.010 & 0.012 & 0.006 & & & \\
\hline
\end{tabular}

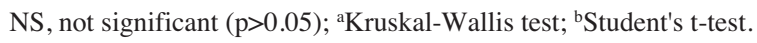

hydatidiform mole developed PTD after evacuation for which either single-agent chemotherapy (MTX, n=7), or single-agent followed by multi-agent chemotherapy (EMA$\mathrm{CO}, \mathrm{n}=6$ ) was indicated.

Immunoassays. The measurements of total hCG (i.e., intact $\mathrm{hCG}$ and free $\beta$-subunit, $\mathrm{hCG}+\beta)$ in serum were performed with a sensitive and specific radioimmunoassay (RIA) that was developed in our laboratory and described previously (12). The RIA used a polyclonal anti-rabbit antiserum. A highly purified hCG $\beta$-subunit preparation labeled with Iodine-125 ( $\mathrm{NaI}^{125}$, Amersham plc, Amersham, England) was used as a tracer. The RIA was calibrated with the third International Standard Preparations for intact hCG (WHO third IS hCG 75/537, obtained from the National Institute for Biological Standards, Potters Bar, England). The measuring range for the standard dose-response curve of the $\mathrm{hCG}+\beta$ RIA was $0.027-2.14 \mathrm{nmol} / \mathrm{l}$ (equivalent to 9.29-743 IU/l). The intra- and inter-assay coefficients of variation $\left(\mathrm{CV}_{\mathrm{w}}\right.$, $\mathrm{CV}_{\mathrm{b}}$ ) for means of duplicate measurements for two serum pools [mean: $0.267 \mathrm{nmol} / 1$ (93 IU/l) and $1.50 \mathrm{nmol} / 1$ (520 IU/1)] were $7.3 \%$ and $12 \%$. The hCG+ß RIA cross-reacted $100 \%$ with intact hCG and $1000 \%$ with hCGß. The 95th percentile of the reference interval of healthy control pregnancies for the $\mathrm{hCG}+\beta$ assay was established at 0.053 nmol/l (18.6 IU/l of the WHO third IS hCG 75/537) (12).

ITA was measured with the Nichols Advantage $\AA$ invasive trophoblast antigen assay (Nichols Institute Diagnostics, San Clemente, CA, USA), and the assay was performed according to the instructions provided by the manufacturer. The ITA assay is a two-step, two-site immuno-chemiluminometric assay that uses two monoclonal antibodies. The capture antibody (B152) is biotin labeled, while the second antibody (B207) is labeled with acridinium ester and used for detection. The analytical sensitivity of the assay is $<0.005$ $\mathrm{nmol} / \mathrm{l}(<0.2 \mu \mathrm{g} / \mathrm{l})$. Cross reactivity as documented by the supplier, is $<1 \%$ with recombinant hCG (Sigma C6322), $5.4 \%$ with nicked hCG, $4.5 \%$ with non-nicked hCG, $1.5 \%$ with nicked free $\beta \mathrm{hCG}$, and $1.0 \%$ with non-nicked free ßhCG.

Statistical analysis. Both $\mathrm{hCG}+\mathrm{B}$ and ITA concentrations were determined for the same serum samples. The half-lives for $\mathrm{hCG}+\beta$ and ITA were calculated for each individual patient from linear regression analysis of log-transformed concentrations against time. The first time-point preceding the final phase of decreasing concentrations was taken as the starting point. In the patients with persistent disease this coincided with the start of the medication. Except for the first one encountered, values below the detection limits were excluded from calculation. The half-lives were obtained for 7 patients with uneventful regression, and 13 patients with PTD, of whom 7 received mono-chemotherapy and 6 monochemotherapy followed by multi-chemotherapy. The halflives of $\mathrm{hCG}+\beta$ and ITA were compared by Paired t-test within the three subgroups. Between the subgroups, the half-lifes of $\mathrm{hCG}+\beta$ and ITA, were compared by the Kruskal-Wallis test (SPSS, version 12.1).

\section{Results}

Table I shows the characteristics of the assay results and chemotherapy courses of 7 patients with uneventful regression (group 1), and of patients with PTD treated either with MTX (group 2, n=7) or with EMA-CO (group 3, n=6). The total observation periods of the patients comprising groups 1, 2 and 3, respectively, ranged between 11-95, 1393, and 38-129 weeks. During these follow-up periods, the total number of $\mathrm{hCG}+\beta$ and ITA measurements ranged between 9-17 and 6-12 in group 1, between 9-23 and 6-10 for group 2, and between 17-37 and 13-32 for group 3. Table I also shows that the observed maximum concentrations of the two analytes tested increased in the order uneventful regression (group 1), single agent (group 2), multi-agent chemotherapy (group 3 ). The maximum concentration observed for $\mathrm{hCG}+\beta$ was $5.1 \mathrm{nmol} / \mathrm{l}$ in group $1,75 \mathrm{nmol} / \mathrm{l}$ in group 2, and $590 \mathrm{nmol} / 1$ 

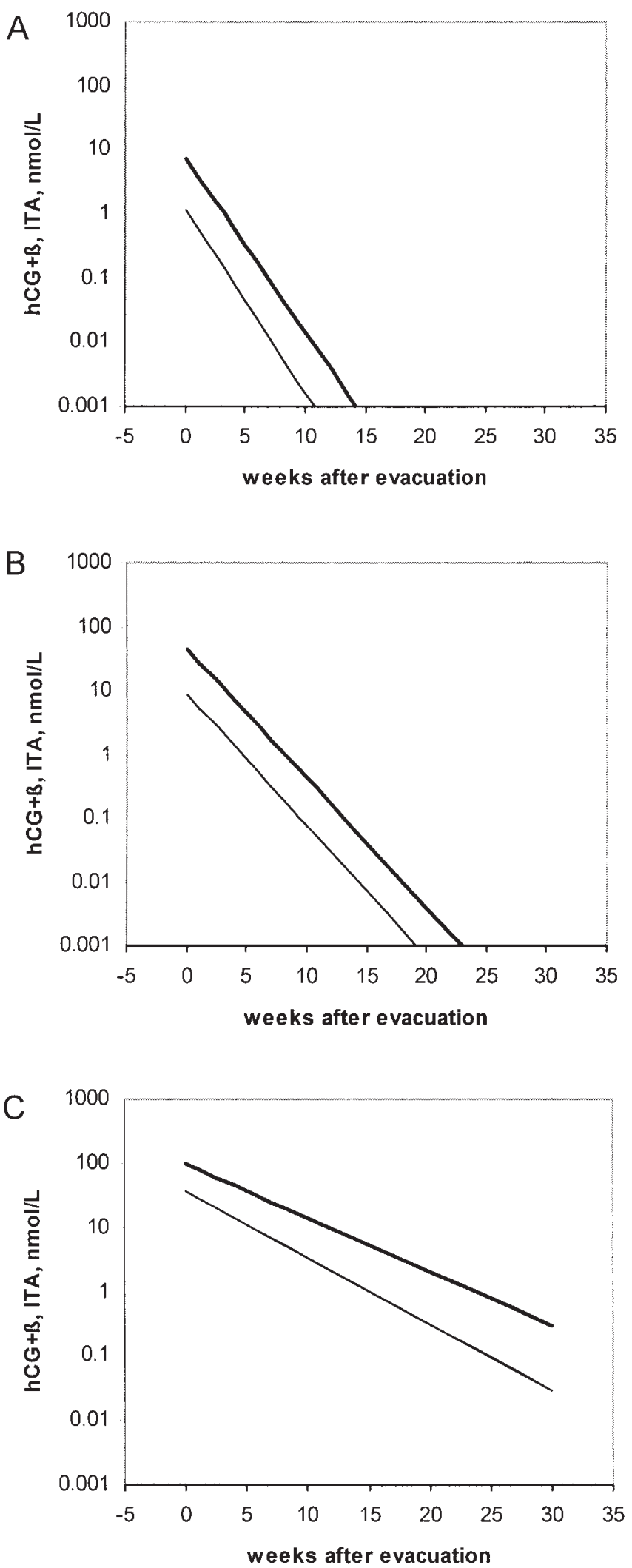

Figure 2. (A) Post molar regression of $\mathrm{hCG}+\beta$ (strong line) and ITA (narrow line) in serum; spontaneous regression (group 1); (B) PTD treated with MTX (group 2); (C) PTD treated with MTX and EMA-CO (group 3).

in group 3. For ITA, these figures were $1.1 \mathrm{nmol} / \mathrm{l}$ in group 1 , $45 \mathrm{nmol} / \mathrm{l}$ in group 2 , and $110 \mathrm{nmol} / \mathrm{l}$ in group 3 .
The number of MTX courses given to the patients in group 2 ranged between 3-9 courses given throughout post evacuation weeks 3 and 19. The 6 patients comprising group 3 received between 6-10 courses of MTX which were given 3-23 weeks after evacuation of the molar pregnancy. Next to MTX treatment, group 3 patients received multi agent chemotherapy in courses ranging between 1-5 that were given in weeks 20-34 after evacuation of their mole. Fig. 1 depicts the time course of $h C G+\beta$ and ITA regression of two typical examples of each group.

Fig. 2 depicts the mean time course of $\mathrm{hCG}+\beta$ and ITA concentrations as obtained for the three groups. The mean semi-logarithmic regression lines for either analyte in the case of uneventful regression (group 1) are given in Fig. 2A, while those for the patients receiving mono-chemotherapy (group 2), or mono-chemotherapy followed by polychemotherapy (group 3) are given in Fig. 2B and C, respectively. Based on these data, we calculated the mean serum half-lives (disappearance rates) for the two analytes (Table II). Significant 3- to 4-fold increases of mean serum half-lives for either parameter was observed in group 3 as compared with group 1 (both $\mathrm{p}=0.003$ ) or with group 2 (both $\mathrm{p}=0.003$ ). No significant differences of mean serum half-life for either parameter were observed between group 1 and 2 . Significantly shorter mean half-lives for ITA than those calculated for $\mathrm{hCG}+\beta$ were observed in the three groups of patients. The mean ratios of ITA $/ \mathrm{hCG}+\beta$ (given in $\mathrm{mol} /$ mol\%) of groups 1, 2 and 3 were 29.3, 28.2 and $35.4 \mathrm{~mol} /$ mol\%, respectively, and did not differ significantly between the three groups tested.

\section{Discussion}

The aim of the present study was to compare ITA with $\mathrm{hCG}+\beta$ measurements in serum in the course of uneventful regression after evacuation of a hydatidiform mole as well as throughout the follow-up period of mono-, or mono- and poly-chemotherapy treatment in cases where persistent trophoblastic disease occurred after evacuation of the molar pregnancy. Disappearance rates of the two analytes were calculated for the three groups of patients and the resulting half-lives were compared between the three groups. The mean serum half-lives for the two parameters were significantly 3 to 4 times longer in the group receiving mono- and polychemotherapy as compared to the groups with either monochemotherapy or uneventful regression, while no significant differences were observed between the group with monochemotherapy and the one with uneventful regression. Significantly shorter mean half-lives for ITA than those calculated for $\mathrm{hCG}+\beta$ were observed in all three groups of patients.

The etermination of disappearance rates for $\mathrm{hCG}+\beta$ and ITA revealed that the half-life of $\mathrm{hCG}+\beta$ in the circulation was significantly longer than that of ITA, irrespective of therapy. The longer half-life for $\mathrm{hCG}+\beta$ may be due to the rather complex multi-exponential decay of intact hCG (median half-times between 3.6-53 h) and free hCG $\beta$ subunit (1-194 h) as reported in the serum of six women with term pregnancies throughout a period of 21 days after delivery (13). Although the data are not fully comparable with those presented here for the disappearance of $\mathrm{hCG}+ß$ during 
follow-up and treatment of PTD, these data indicate that the presence of various molecular forms of hCG, all with different half-life times, may affect the observed disappearance rates of total hCG immunoreactivity as measured with our hCG+ $\beta$ RIA. Such data on half-lives of ITA are not yet available.

The different degrees of (hyper)glycosylation of hCG and ITA may also contribute to the differences in the disappearance rates of these analytes. About one-third of the molecular mass of hCG consists of carbohydrates attached to six glycosylation sites on hCGß and two on hCG $\alpha$. The carbohydrate chains contain 8-15 terminal sialic acids, and therefore, hCG displays extensive heterogeneity (isoforms) (14). It has been reported that hyperglycosylated hCG can vary greatly in sialic acid content, with molecules having between $0-19$ sialic acid residues $(7,15,16)$. Hyperglycosylated hCG generally contains less of these acidic sugars than regular hCG (7). As terminal sialic acid protects the galactose residues of the carbohydrate chains against metabolic degradation by neuraminidases (17-19), and hCG has a higher sialic acid content than ITA, regular hCG might be better protected against metabolic breakdown than ITA resulting in a longer half-life in the circulation of hCG than of ITA. We also observed significantly longer half-lives of $\mathrm{hCG}+\beta$ or ITA in the PTD group treated with polychemotherapy (group 3) as compared with the two other groups. The presence of severely persistent trophoblastic tissue in these patients unequivocally requiring multi-agent chemotherapeutic treatment may have been responsible for this. Similar disappearance rates for $\mathrm{hCG}+\beta$ and ITA were found in the group with uneventful, spontaneously regressing trophoblast, and in the PTD group responding to monochemotherapy. Likewise, the time needed for complete regression to normal serum concentrations were also similar. Thus, the PTDs curable with mono-chemotherapy could be designated as 'moderate persistent trophoblasts', and those requiring poly-chemotherapy as 'severe persistent trophoblasts'.

The question whether ITA is a better marker of PTD than $\mathrm{hCG}+\beta$ parallels the discussion by Okamoto et al (20) regarding the usefulness of hCG $\beta$-core fragment (hCGßcf) for early prediction of subsequent development of post-molar PTD. These authors reported that, due to its short half-life, serum hCGßcf rapidly declined and became undetectable after evacuation of the mole with subsequent spontaneous resolution. A similar observation was obtained in the present study for ITA. Okamoto et al further argued that after evacuation, serum hCGßcf remained elevated or started to rise before PTD was diagnosed based on the rise of hCG serum levels. They concluded that hCGßcf in the serum after mole evacuation may indicate persistence of viable trophoblasts more sensitive than hCG. Our present data are too limited to merit whether the same conclusion would hold for ITA. Okamoto et al further reported that, once chemotherapy started, hCGßcf became more rapidly undetectable than hCG because of its relatively low levels in serum and the more rapid clearance from the circulation than hCG. Therefore, it seems that hCGßcf may not be suitable for the follow-up of patients receiving chemotherapy.

The significance of persistent low levels of hCG in the serum of non-pregnant patients without clinical evidence of
PTD and its relationship with ITA was studied by Khanlian et al (11). These authors concluded that either the absence of detectable ITA, i.e., serum concentrations for ITA $<0.005 \mathrm{nmol}$ $(<0.2 \mu \mathrm{g} / \mathrm{l})$, or the presence of insignificant amounts of ITA (accounting for $<5 \%$ of the total hCG immunoreactivity determined with their total hCG assay) were indicative for the absence of invasive cytotrophoblastic cells and that 'quiescent PTD' was present. Because low, but significantly increased hCG serum concentrations were present, these patients were treated, but despite treatment, the low hCG levels persisted. In 4 out of 38 cases, the low hCG concentrations suddenly rose sharply, and the proportion of ITA in such samples increased to $>80 \%$ and up to $100 \%$ of the total circulating hCG. Similarly, this was also observed in specimens from 15 other cases with proven GTD (choriocarcinoma or placental site trophoblastic tumor) $(10,11)$. In the present study, we observed sharply decreasing hCG+ß and ITA concentrations in the post evacuation follow-up of patients with uneventful regression, and in PTD treated with mono-, or mono- followed by poly-chemotherapy, respectively, although the profiles of the serum ITA regression curves compared with those of total hCG $+\beta$ immuno-reactivity did not seem to differ in the various treatment groups tested. Thus, it may be questioned whether the absence of invasive trophoblastic disease (group 1), or the presence of this disease with proven response to chemotherapy treatment (groups 2 and 3 ) is accurately reflected by the proportion of ITA accounting for the total hCG immunoreactivity. In this respect, it would be of interest to investigate in a prospective randomised setting the effect of additional chemotherapy courses during the treatment at the time point where serum ITA concentrations become non-detectable whereas $\mathrm{hCG}+\beta$ determinations are not yet normalised, but are still declining and significantly above the detection limit of the assay.

In conclusion, we have studied a series of patients with uneventful post-molar $\mathrm{hCG}+\beta$ regression in a longitudinal setting and compared these results with those of ITA. Similar comparisons were made in groups of patients who developed post-molar PTD and normalised upon treatment with either mono-, or mono- and subsequent poly-chemotherapeutic agents. The serum half-lives were calculated and revealed lower disappearance rates for $\mathrm{hCG}+\beta$ than for ITA. Multichemotherapy treatment of post molar PTD displayed significantly a longer half-life than the mono-chemotherapy treatment or spontaneous regression. The implication and the possible clinical value of the more rapid regression of ITA to baseline levels as compared to $\mathrm{hCG}+\beta$ remain to be investigated prospectively.

\section{Acknowledgements}

The authors acknowledge Rob van der Steen for expert technical assistance and Dr Rudolf Schemer, Nichols Institute Diagnostika $\mathrm{GmbH}$, Germany, for providing the invasive trophoblast antigen testing kits.

\section{References}

1. Yedema KA, Verheijen RH, Kenemans P, Schijf CP, Borm GF, Segers MF and Thomas CM: Identification of patients with persistent trophoblastic disease by means of a normal human chorionic gonadotropin regression curve. Am J Obstet Gynecol 168: 787-792, 1993 
2. Khazaeli MB, Hedayat MM, Hatch KD, To AC, Soong SJ, Shingleton HM, Boots LR and LoBuglio AF: Radioimmunoassay of free beta-subunit of human chorionic gonadotropin as a prognostic test for persistent trophoblastic disease in molar pregnancy. Am J Obstet Gynecol 155: 320-324, 1986.

3. Berkowitz RS, Goldstein DP and Bernstein MR: Natural history of partial molar pregnancy. Obstet Gynecol 66: 677-681, 1985.

4. Szulman AE and Surti U: The clinicopathologic profile of the partial hydatidiform mole. Obstet Gynecol 59: 597-602, 1982.

5. Ng TY and Wong LC: Diagnosis and management of gestational trophoblastic neoplasia. Best Pract Res Clin Obstet Gynaecol 17: 893-903, 2003

6. Osborne R and Gerulath A: What is the best regimen for lowrisk gestational trophoblastic neoplasia? A review. J Reprod Med 49: 602-616, 2004.

7. Elliott MM, Kardana A, Lustbader JW and Cole LA: Carbohydrate and peptide structure of the alpha- and beta-subunits of human chorionic gonadotropin from normal and aberrant pregnancy and choriocarcinoma. Endocrine 7: 15-32, 1997.

8. Cole LA, Shahabi S, Oz UA, Bahado-Singh RO and Mahoney MJ: Hyperglycosylated human chorionic gonadotropin (invasive trophoblast antigen) immunoassay: A new basis for gestational Down syndrome screening. Clin Chem 45: 2109-2119, 1999.

9. Kovalevskaya G, Genbacev O, Fisher SJ, Caceres E and O'Connor JF: Trophoblast origin of hCG isoforms: cytotrophoblasts are the primary source of choriocarcinoma-like hCG. Mol Cell Endocrinol 194: 147-155, 2002.

10. Cole LA, Shahabi S, Butler SA, Mitchell H, Newlands ES, Behrman HR and Verrill HL: Utility of commonly used commercial human chorionic gonadotropin immunoassays in the diagnosis and management of trophoblastic diseases. Clin Chem 47: 308-315, 2001.

11. Khanlian SA, Smith HO and Cole LA: Persistent low levels of human chorionic gonadotropin: A premalignant gestational trophoblastic disease. Am J Obstet Gynecol 188: 1254-1259, 2003.
12. Thomas CM, Segers MF and Houx PC: Comparison of the analytical characteristics and clinical usefulness in tumour monitoring of fifteen hCG(-beta) immunoassay kits. Ann Clin Biochem 22: 236-246, 1985.

13. Korhonen J, Alfthan H, Ylostalo P, Veldhuis J and Stenman UH: Disappearance of hCG and its alpha- and beta-subunits after term pregnancy. Clin Chem 43: 2155-2163, 1997.

14. Stenman UH, Alfthan H and Hotakainen K: Human chorionic gonadotropin in cancer [review]. Clin Biochem 37: 549-561, 2004.

15. Imamura S, Armstrong EG, Birken S, Cole LA and Canfield RE: Detection of desialylated forms of human chorionic gonadotropin. Clin Chim Acta 163: 339-349, 1987.

16. Nishimura R, Endo Y, Tanabe K, Ashitaka Y and Tojo S: The biochemical properties of urinary human chorionic gonadotropin from the patients with trophoblastic disease. J Endocrinol Invest. 4: 349-358, 1981.

17. Morell AG, Gregoriadis G and Scheinberg ICH: The role of sialic acid in determining the survival of glycoprotiens in the circulation. J Biol Chem 246: 1461-1467, 1971.

18. Hoermann R, Kubota K and Amir SM: Role of subunit sialic acid in hepatic binding, plasma survival rate, and in vivo thyrotropic activity of human chorionic gonadotropin. Thyroid 3: 41-47, 1993.

19. Ashwell G and Harford J: Carbohydrate-specific receptors of the liver. Annu Rev Biochem 51: 531-554, 1982.

20. Okamoto T, Matsuo K, Niu R, Osawa M and Suzuki H: Human chorionic gonadotropin (hCG) beta-core fragment is produced by degradation of hCG or free hCG beta in gestational trophoblastic tumors: a possible marker for early detection of persistent postmolar gestational trophoblastic disease. J Endocrinol 171: 435-443, 2001. 